\title{
On a Biquaternionic Bag Model
}

\author{
V. V. Kravchenko
}

\begin{abstract}
A bstract. There is considered a biquaternionic reformulation of a linear bag model describing the phenomenon of quarks confinement. The problem reduces to a boundary singular integral equation associated with the biquaternionic hyperholomorphic function theory which allows to study the invertibility and Fredholmness of the bag model.
\end{abstract}

Keywords: Bag models, Dirac equation, hyperholomorphic biquaternionic functions

AMS subject classification: Primary $35 \mathrm{~J} \mathrm{55,} 35 \mathrm{Q} 40,31 \mathrm{~B} 10$, secondary 45 F 15, $81 \mathrm{Q} 05$

\section{Introduction}

One of the mathematical possibilities to describe the phenomenon of quarks confinement is to complement the classical Dirac equation with some additional conditions given on the boundary of a domain occupied by a hadron. Considering the most simple linear bag model (see, e.g., [21: Section 2.1]) as an illustration we show its equivalence to a boundary-value problem for the biquaternionic differential operator studied in $[9,11$ 13]. It was shown there that this biquaternionic operator can be regarded as a spatial analogy of the Cauchy-Riemann operator and that the corresponding analogies of the Borel-Pompey formula, the Cauchy integral formula, the Plemelj-Sokhotzkij formulas, the Cauchy integral theorem, the Morera theorem, and some others are obtained. The mentioned equivalence being expressed in terms of a definite bijective mapping of the Dirac bispinors onto a class of biquaternionic functions enables us to apply this generalization of complex analysis to the investigation of quark models of the hadron structure. In this paper we restrict ourselves to some examples of such applications.

\section{Linear bag model}

Being free particles (in the domain occupied by a hadron) with spin $\frac{1}{2}$ quarks, i.e., the corresponding spinor fields $\Phi: \mathbb{R}^{4} \rightarrow \boldsymbol{C}^{4}$ satisfy the Dirac equation

$$
\left(\gamma_{0} \partial_{t}-\sum_{k=1}^{3} \gamma_{k} \partial_{k}+i m\right) \Phi=0
$$

V. V. Kravchenko: Ukrainian Acad. Sci., Odessa Division of Hydroàcoustics, ul. Sovetskoj Armii 3, Odessa 270100, Ukraine 
where $m \in \mathbb{R}, \partial_{t}=\frac{\partial}{\partial t}$ and $\partial_{k}=\frac{\partial}{\partial x_{k}}$, and where the standard Dirac matrices $\gamma_{0}, \ldots, \gamma_{3}$ are defined by

$$
\begin{aligned}
\gamma_{0}=\left(\begin{array}{cccc}
1 & 0 & 0 & 0 \\
0 & 1 & 0 & 0 \\
0 & 0 & -1 & 0 \\
0 & 0 & 0 & -1
\end{array}\right) & \gamma_{1}=\left(\begin{array}{cccc}
0 & 0 & 0 & -1 \\
0 & 0 & -1 & 0 \\
0 & 1 & 0 & 0 \\
1 & 0 & 0 & 0
\end{array}\right) \\
\gamma_{2}=\left(\begin{array}{cccc}
0 & 0 & 0 & i \\
0 & 0 & -i & 0 \\
0 & -i & 0 & 0 \\
i & 0 & 0 & 0
\end{array}\right) & \gamma_{3}=\left(\begin{array}{cccc}
0 & 0 & -1 & 0 \\
0 & 0 & 0 & 1 \\
1 & 0 & 0 & 0 \\
0 & -1 & 0 & 0
\end{array}\right) .
\end{aligned}
$$

Usually, time-harmonic spinor fields $\Phi$ are considered, that is,

$$
\Phi(t, x)=q(x) \mathrm{e}^{\mathrm{i} \omega t}
$$

where $\omega \in \mathbb{R}$ and $q=\left(q_{0}, q_{1}, q_{2}, q_{3}\right)^{\top}: \mathbb{R}^{3} \rightarrow \boldsymbol{C}^{4}$. Then from (1) we obtain for $q$ the equation

$$
\mathbb{D}_{\omega, m} q:=\left(i \omega \gamma_{0}-\sum_{k=1}^{3} \gamma_{k} \partial_{k}+i m\right) q=0
$$

which generally speaking must be satisfied in some domain $\tilde{\Omega} \subset \mathbb{R}^{3}$. We assume $q \in$ $C^{1}(\tilde{\Omega}) \cap C(\overline{\tilde{\Omega}})$ and the boundary $\partial \tilde{\Omega}$ to be a closed Liapunov surface in $\mathbb{R}^{3}$ which can be closed by an infinite point.

The confinement of quarks to the domain $\tilde{\Omega}$ is described by the boundary condition

$$
\sum_{k=1}^{3} \gamma_{k} \tilde{n}_{k} q(x)=i q(x) \quad(x \in \partial \tilde{\Omega})
$$

where $\tilde{n}_{k}$ are the components of the unit outward normal to the boundary $\partial \tilde{\Omega}$. The boundary condition (3) imposes a ban on the particle flow through the surface of the confining region.

The equations (2) and (3) form the so-called linear bag model (see [21: Section 2.1]). Some particular solutions of problem (2), (3) have been obtained for the case $\tilde{\Omega}$ to be a ball (see, e.g., [21], [4: p. 386]) as well as for the case when $\tilde{\Omega}$ is a domain contained between two parallel hyperplanes (see [17: p. 58]).

In order to obtain a biquaternionic formulation of the model (2), (3) first of all one should construct a biquaternionic analog of the Dirac equation, that is - in correspondence with an old problem posed by Sommerfeld - to rewrite the Dirac equation (1) in a form, where the rank of the algebra of involved matrices coincides with the number of components of the wave function. A review of results in this direction one can find in [1: Chapter 4]. In [2] an approach which is near to the methods of the present paper was used, and it was shown that in this case the symmetrical analysis of the Dirac equation becomes simpler.

We construct a biquaternionic analog of the Dirac equation in Section 4 . 


\section{Some necessary biquaternionic definitions and results}

Let us denote by $\mathbb{H}(\boldsymbol{C})$ the algebra of complex quaternions (biquaternions), i.e.

$$
\mathbb{H}(\boldsymbol{C})=\left\{\alpha=\sum_{k=0}^{3} \alpha_{k} i_{k}: \alpha_{k} \in \boldsymbol{C}(k=0,1,2,3)\right\}
$$

where $i_{k}$ are the standard basic quaternions. By definition for the imaginary unit $i$ from $C$ the relations $i i_{k}=i_{k} i$ are true. We let

$$
\vec{\alpha}=\operatorname{Vec} \alpha=\sum_{k=1}^{3} \alpha_{k} i_{k}
$$

and identify purely imaginary biquaternions (i.e. such $\alpha=\left(\alpha_{0}, \alpha_{1}, \alpha_{2}, \alpha_{3}\right) \in \mathbb{H}(\boldsymbol{C})$ that Sc $\alpha:=\alpha_{0}=0$ ) with vectors from $\boldsymbol{C}^{3}$. We will be in need of the different conjugations

$$
\bar{\alpha}=\operatorname{Sc} \alpha-\operatorname{Vec} \alpha
$$

and

$$
\alpha^{*}:=C(\alpha)=\operatorname{Re} \alpha-i \operatorname{Im} \alpha=\sum_{k=0}^{3}\left(\operatorname{Re} \alpha_{k}-i \operatorname{Im} \alpha_{k}\right) i_{k}
$$

The algebra $I H(\boldsymbol{C})$ contains the subset $\mathcal{S}$ of zero divisors. Remark that

$$
\mathcal{S}=\{\alpha \in H(\boldsymbol{C}) \mid \alpha \neq 0 \text { and } \alpha \bar{\alpha}=0\}
$$

On $H(\boldsymbol{C})$-valued functions

$$
f(x)=\sum_{k=0}^{3} f_{k}(x) i_{k} \quad\left(x \in \Omega \subset \mathbb{R}^{3}, f_{k} \in C^{1}(\Omega)\right)
$$

the operator

$$
D_{\alpha}=D+M^{\alpha} \quad \text { with } D=\sum_{k=1}^{3} i_{k} \partial_{k} \quad \text { and } M^{\alpha} f=f \alpha \quad(\alpha \in \mathbb{H}(\boldsymbol{C}))
$$

is defined where $D$ is the so-called Moisil-Theodoresco operator. In what follows it is sufficient to suppose that the domain $\Omega$ is obtained from $\tilde{\Omega}$ by the reflection $x_{3} \rightarrow-x_{3}$. Then, the restrictions on $\Omega$ coincide with the above restrictions on $\tilde{\Omega}$.

A function $f \in \operatorname{Ker} D_{\alpha}(\Omega)$ is called $\alpha$-hyperholomorphic in $\Omega$. The equation

$$
D_{\alpha} f=0
$$

can be rewritten in the equivalent vector form

$$
\begin{array}{r}
\alpha_{0} f_{0}-\operatorname{div} \vec{f}-\langle\vec{f}, \vec{\alpha}\rangle=0 \\
\operatorname{grad} f_{0}+\operatorname{rot} \vec{f}+[\vec{f} \times \vec{\alpha}]+f_{0} \vec{\alpha}+\alpha_{0} \vec{f}=0
\end{array}
$$


where the brackets $\langle\cdot, \cdot\rangle$ and $[\cdot, \cdot]$ denote the scalar and vector product, respectively.

When $\alpha=0,(5)$ is the well-known Moisil-Theodoresco system studied by many authors (see, e.g., [3: p.220], [5: p. 323] and [20]). The case $\alpha_{0} \in \mathbb{R}$ and $\vec{\alpha}=0$ was considered in [6: Section 4.2]. When $\alpha_{0}=0$ and $\alpha_{1}, \alpha_{2}, \alpha_{3} \in \mathbb{R}$ the system (5) defines generalized holomorphic vectors studied in $[7,9,16,18,19]$.

The general case $\alpha \in \mathbb{H}(\boldsymbol{C})$ was studied in [11 - 13]. It was shown that equation (4) can be regarded as a spatial analog of the Cauchy-Riemann equations, and the analogs of the Borel-Pompey formula, the Cauchy integral formula, the Plemelj-Sokhotzkij formulas, the Cauchy integral theorem, the Morera theorem, and some others are obtained. Here we only fomulate some results (Theorem 1 and Theorem 2) from [11 - 13] which are necessary for what follows.

Let

$$
\vartheta_{\nu}(x):=-\frac{1}{4 \pi|x|} \mathrm{e}^{-\mathrm{i} \nu|x|} \quad(\nu \in \boldsymbol{C})
$$

Then the functions

$$
\mathcal{K}_{ \pm \nu}(x)=-\left(D_{\mp \nu} \vartheta_{\nu}\right)(x)=\vartheta_{\nu}(x)\left( \pm \nu+\frac{x}{|x|^{2}}+i \nu \frac{x}{|x|}\right)
$$

where $x=\sum_{k=1}^{3} x_{k} i_{k}$ are fundamental solutions to the operators $D_{ \pm \nu}$. Let us introduce the integral operators

$$
\left(T_{\nu} f\right)(x)=\int_{\Omega} \mathcal{K}_{\nu}(x-y) f(y) d \Omega_{y} \quad\left(x \in \mathbb{R}^{3}\right)
$$

and

$$
\left(K_{\nu} f\right)(x)=-\int_{\Gamma} \mathcal{K}_{\nu}(x-y) \vec{n}(y) f(y) d \Gamma_{y} \quad\left(x \in \mathbb{R}^{3} \backslash \Gamma\right)
$$

which are the analogs of the complex $T$-operator and Cauchy type operator corresponding to $D_{\nu}(\nu \in \boldsymbol{C})$. Here $\Gamma=\partial \Omega$ and $\vec{n}=\sum_{k=1}^{3} n_{k} i_{k}$ is the unit outward normal to $\Gamma$. Using (6), for an arbitrary element $\alpha \in \mathbb{H}(\boldsymbol{C})$ the corresponding operator $T_{\alpha}$ is defined by

$$
T_{\alpha}= \begin{cases}\Pi^{+} T_{\xi}+\Pi^{-} T_{\zeta} & \text { for } \alpha \notin \mathcal{S} \text { with } \vec{\alpha}^{2} \neq 0 \\ T_{\alpha_{0}}+M^{\vec{\alpha}} \frac{\partial}{\partial \alpha_{0}}\left[T_{\alpha_{0}}\right] & \text { for } \alpha \notin \mathcal{S} \text { with } \vec{\alpha}^{2}=0 \\ \Pi^{+} T_{2 \alpha_{0}}+\Pi^{-} T_{0} & \text { for } \alpha \in \mathcal{S} \text { with } \alpha_{0} \neq 0 \\ T_{0}+M^{\alpha} W_{0} & \text { for } \alpha \in \mathcal{S} \text { with } \alpha_{0}=0\end{cases}
$$

where

$$
\Pi^{ \pm}=\frac{1}{2 \gamma} M^{(\gamma \pm \vec{\alpha})} \quad(\gamma \in \boldsymbol{C}), \quad \gamma^{2}=\vec{\alpha}^{2}, \quad \xi=\alpha_{0}+\gamma, \quad \zeta=\alpha_{0}-\gamma
$$

and

$$
\left(W_{\mu} f\right)(x)=\int_{\Omega} \vartheta_{\mu}(x-y) f(y) d \Omega_{y} \quad \therefore\left(\mu \in \boldsymbol{C}, x \in \mathbb{R}^{3}\right)
$$


(see [11] and [13]). Analogously, using (7); for an arbitrary element $\alpha \in \mathbb{H}(\boldsymbol{C})$ the corresponding operator $K_{\alpha}$ is defined by the formula

$$
K_{\alpha}= \begin{cases}\Pi^{+} K_{\xi}+\Pi^{-} K_{\zeta} & \text { for } \alpha \notin \mathcal{S} \text { with } \vec{\alpha}^{2} \neq 0 \\ K_{\alpha_{0}}+M^{\vec{\alpha}} \frac{\partial}{\partial \alpha_{0}}\left[K_{\alpha_{0}}\right] & \text { for } \alpha \notin \mathcal{S} \text { with } \vec{\alpha}^{2}=0 \\ \Pi^{+} K_{2 \alpha_{0}}+\Pi^{-} K_{0} & \text { for } \alpha \in \mathcal{S} \text { with } \alpha_{0} \neq 0 \\ K_{0}-M^{\alpha} V_{0} & \text { for } \alpha \in \mathcal{S} \text { with } \alpha_{0}=0\end{cases}
$$

where

$$
\left(V_{\mu} f\right)(x)=\int_{\Gamma} \vartheta_{\mu}(x-y) \vec{n}(y) f(y) d \Gamma_{y} .
$$

Remark that $\Pi^{ \pm}$are mutually complementary projection operators.

Let us introduce the operators

$$
P_{\alpha}=\frac{1}{2}\left(I+S_{\alpha}\right) \quad \text { and } \quad Q_{\alpha}=I-P_{\alpha}
$$

where $I$ is the identity operator and

$$
\left(S_{\alpha} f\right)(x)=2\left(K_{\alpha} f\right)(x) \quad(x \in \Gamma)
$$

Here the integrals exist in the sense of Cauchy principal values. Now we have the following

Theorem 1: The following statements are true:

1. (The Borel-Pompey formula) Let $f \in C^{1}(\Omega) \cap C(\bar{\Omega})$. Then

$$
\left(K_{\alpha} f\right)(x)+\left(T_{\alpha} D_{\alpha} f\right)(x)=f(x)
$$

for all $x \in \Omega$.

2. (The Cauchy integral formula) Let $f \in C^{1}(\Omega) \cap C(\bar{\Omega}) \cap \operatorname{Ker} D_{\alpha}(\Omega)$.' Then $K_{\alpha} f=f$ in $\Omega$.

3. (The Plemelj-Sokhotzkij formulas) Let $f \in C^{0, e}(\Gamma)(0<\varepsilon \leq 1)$. Then

$$
\lim _{\substack{x \in y \in \Gamma \\ x \in \Omega}}\left(K_{\alpha} f\right)(x)=\left(P_{\alpha} f\right)(y) \quad \text { and } \quad \lim _{\substack{x \rightarrow y \in \Gamma \\ x \in \Omega}}\left(K_{\alpha} f\right)(x)=\left(-Q_{\alpha} f\right)(y) \text {. }
$$

for any $y \in \Gamma$.

4. (Involutiveness of the operator of singular integration) Let $f \in C^{0, c}(\Gamma) \quad(0<$ $\varepsilon \leq 1)$. Then $S_{\alpha}^{2} f=f$.

5. Let $f \in C^{1}(\Omega) \cap C(\bar{\Omega})$. Then $D_{\alpha} T_{\alpha} f=f$ in $\Omega$.

6. (Cauchy integral theorem) Let $f \in C^{1}(\bar{\Omega}) \cap \operatorname{Ker} D_{\alpha}(\bar{\Omega})$. Then

$$
\int_{\Gamma} \vec{n}(y) f(y) d \Gamma_{y}+\int_{\Omega} f(y) \alpha d \Omega_{y}=0 .
$$

7. (The Morera theorem) Let $f^{\prime} \in C^{1}(\Omega)$ and $D_{\alpha} f \in L_{p}(\Omega)(p>1)$. If for any Liapunov manifold without boundary $s=\partial \Omega_{S} \subset \Omega$

$$
\int_{s} \vec{n} f d s=-\int_{\Omega_{s}} f \alpha d \Omega_{S},
$$

then $f$ is $\alpha$-hyperholomorphic in $\Omega$.

From the statements 2 and 3 of Theorem 1 there follows 
Theorem 2: A function $f: \Gamma \rightarrow I H(C)$ satisfying the Holder condition is the boundary value of a solution $g$ of equation (4) in $\Omega$ if and only if $P_{\alpha} f=f$ on $\Gamma$ : Moreover, if $P_{\alpha} f=f$ on $\Gamma$ is satisfied, then $g=K_{\alpha} f$ is the.above mentioned solution of equation (4).

\section{Biquaternionic analog of the Dirac equation (1)}

Let us introduce a mapping $\mathcal{A}$ which transforms a function $\Phi: \tilde{G} \subset \mathbb{R}^{4} \rightarrow C^{4}$ to a function $F=\mathcal{A}[\Phi]: G \subset \mathbb{R}^{i} \rightarrow \mathbb{H}(\boldsymbol{C})$ by the rule

$$
\begin{aligned}
F= & \left(\operatorname{Re} \tilde{\Phi}_{0}+i \operatorname{Im} \tilde{\Phi}_{2}\right) i_{0}+\left(\operatorname{Im} \tilde{\Phi}_{1}-i \operatorname{Re} \tilde{\Phi}_{3}\right) i_{1} \\
& -\left(\operatorname{Re} \tilde{\Phi}_{1}+i \operatorname{Im} \tilde{\Phi}_{3}\right) i_{2}-\left(\operatorname{Im} \tilde{\Phi}_{0}-i \operatorname{Re} \tilde{\Phi}_{2}\right) i_{3}
\end{aligned}
$$

where $\tilde{\Phi}=\Phi\left(t, x_{1}, x_{2},-x_{3}\right)$. Then

$$
\begin{array}{r}
\mathcal{A}^{-1}(F)=\left(\operatorname{Re} \tilde{F}_{0}-i \operatorname{Re} \tilde{F}_{3},-\operatorname{Re} \tilde{F}_{2}+i \operatorname{Re} \tilde{F}_{1}\right. \\
\left.\operatorname{Im} \tilde{F}_{3}+i \operatorname{Im} \tilde{F}_{0},-\operatorname{Im} \tilde{F}_{1}-i \operatorname{Im} \tilde{F}_{2}\right)^{\top} .
\end{array}
$$

The following lemma gives some arithmetical properties of the mapping $\mathcal{A}$.

Lemma 1 (see [10]): Let $\Phi: \tilde{G} \subset \mathbb{R}^{4} \rightarrow \boldsymbol{C}^{4}$ be some function. Then the following statements are true:

1. $\mathcal{A}\left(\gamma_{0} \Phi\right)=(\mathcal{A}(\Phi))^{*}$

2. $\mathcal{A}\left(\gamma_{0} \gamma_{1} \Phi\right)=i i_{1} \mathcal{A}(\Phi), \mathcal{A}\left(\gamma_{0} \gamma_{2} \Phi\right)=i i_{2} \mathcal{A}(\Phi)$ and $\dot{\mathcal{A}}\left(\gamma_{o} \gamma_{3} \Phi\right)=-i i_{3} \mathcal{A}(\Phi)$

3. $\mathcal{A}(i \Phi)=-\mathcal{A}(\Phi) i_{3}$ and $\mathcal{A}\left(\gamma_{5} \Phi\right)=i \mathcal{A}(\Phi) i_{3}$ where $\gamma_{5}=-i \gamma_{0} \gamma_{1} \gamma_{2} \gamma_{3}$.

Let us consider the biquaternionic equation

$$
N F:=\left(i \partial_{t}+D-m i C M^{i_{3}}\right) F=0
$$

Remember that $C$ is the conjugation operator defined by $C(a)=\operatorname{Re} a-i \operatorname{Im} a, D=$ $\sum_{k=1}^{3} i_{k} \partial_{k}$ is the Moisil-Theodoresco operator and the operator $M^{i_{3}}$ is defined by $M^{i_{3}} f=f i_{3}$.

Theorem 3 (see [10]): Between the solutions of the Dirac equation (1) and the solutions of the biquaternionic equation (8) there exists a one-to-one correspondence given by the bijection $\mathcal{A}$, namely, if $\Phi$ is a solution of equation (i), then $F=\mathcal{A}[\Phi] \in$ Ker $N$ and, vice versa, if $F$ is a solution of equation (8), then $\Phi=\mathcal{A}^{-1}[F]$ is a solution of equation (1).

Referring for the proof of the theorem to [10] we remark that it can be done by a straightforward calculation. 
The operator $N$ defined by (8) is not convenient for further investigations because of the presence of the conjugation operator $C$. In [10] the most radical method, for deliverance from it was used considering only the massless spinor fields corresponding to the neitrino. Here, having in mind the massive quarks, we cannot permit ourselves such a simple way. Thus, the most urgent problem is to remove the conjugation operator $\dot{C}$ differently.

The first step is based on the well-known matrix equality (see, e.g., [14: p. 88] and [15: p. 398])

$$
\left(\begin{array}{cc}
I & C \\
I & -C
\end{array}\right)\left(\begin{array}{cc}
A & B \\
C B C & C A C
\end{array}\right)\left(\begin{array}{cc}
I & I \\
C & -C
\end{array}\right)=2\left(\begin{array}{cc}
A+B C & 0 \\
0 & A-B C
\end{array}\right)
$$

where $A$ and $B$ are some linear operators and $C$ is a linear operator with $C^{2}=I$. Let us take

$$
A=i \partial_{t}+D \quad \text { and } \quad B=-m i M^{i_{3}} .
$$

Then denoting $N^{\prime}=i \partial_{t}+D+m i C M^{i_{3}}$ we have

$$
\left(\begin{array}{cc}
N & 0 \\
0 & N^{\prime}
\end{array}\right)=\frac{1}{2}\left(\begin{array}{cc}
I & C \\
I & -C
\end{array}\right)\left(\begin{array}{cc}
i \partial_{t}+D & -m i M^{i_{3}} \\
m i M^{i_{3}} & -i \partial_{t}+D
\end{array}\right)\left(\begin{array}{cc}
I & I \\
C & -C
\end{array}\right) .
$$

That is, if $F \in \operatorname{Ker} N$ and $G \in \operatorname{Ker} N^{\prime}$, then the functions $f=\frac{1}{2}(F+G)$ and $g=$ $\frac{1}{2}\left(F^{*}-G^{*}\right)$ satisfy the system

$$
\begin{array}{r}
\left(i \partial_{t}+D\right) f-m i g i_{3}=0 \\
\left(-i \partial_{\ell}+D\right) g+m i f i_{3}=0 .
\end{array}
$$

Remark that $F=f+g^{*}$ and $G=f-g^{*}$

For the second step we introduce the notation

$$
P_{k}^{ \pm}=\frac{1}{2} M^{1 \pm i i_{k}} \quad(k=1,2,3)
$$

Any pair of operators $P_{k}^{+}$and $P_{k}^{-}$with a fixed $k$ is a pair of mutually complementary projectors on the set of $I H(C)$-valued functions. Let us apply the operators $P_{1}^{+}$and $P_{1}^{-}$ to the first and second equality of system (9), respectively. Then, taking into account the commutation property $P_{1}^{ \pm} M^{i_{3}}=M^{i_{3}} P_{1}^{\mp}$, we obtain the system

$$
\begin{aligned}
& \left(i \partial_{t}+D\right) P_{1}^{+} f-m i M^{i_{3}} P_{1}^{-} g=0 \\
& \left(i \partial_{t}-D\right) P_{1}^{-} g-m i M^{i s} P_{1}^{+} f=0 .
\end{aligned}
$$

Let us add and subtract both equalities of this system. Then for the functions

$$
\varphi=P_{1}^{+} f-P_{1}^{-} g \quad \text { and } \quad \psi=P_{1}^{-} f-P_{1}^{+} g
$$

we have two separate equations:

$$
\begin{aligned}
& P_{1}^{+}\left(i \partial_{t}+D\right) \varphi+P_{1}^{-}\left(-i \partial_{t}+D\right) \varphi-m \dot{\varphi} i_{2}=0 \\
& P_{1}^{+}\left(-i \partial_{t}+D\right) \psi+P_{1}^{-}\left(i \partial_{t}+D\right) \psi+m \psi i_{2}=0
\end{aligned}
$$


Remark that $f=P_{1}^{+} \varphi+P_{1}^{-} \psi$ and $g=-P_{1}^{+} \psi-P_{1}^{-} \varphi$. Let us remark further that the equations (11) and (12) are not independent. Indeed, if $\varphi$ is any solution of equation (11), then the function $\psi:=\varphi i i_{3}=\left(P_{3}^{+}-P_{3}^{-}\right) \varphi$ is a solution of equation (12) and vice versa:

The last step consists in returning from the function $\varphi$ satisfying equation (11) to the function $F$ satisfying equation (8), because (11) is the required biquaternionic equation which is equivalent to equation (8) and does not contain the conjugation operator $C$. Thus

$$
\begin{aligned}
F=f+g^{*} & =P_{1}^{+} \varphi+P_{1}^{-}\left(\varphi i i_{3}\right)-C\left(P_{1}^{+}\left(\varphi i i_{3}\right)+P_{1}^{-} \varphi\right) \\
& =\left(P_{1}^{+}-P_{1}^{+} C+P_{1}^{-}\left(P_{3}^{+}-P_{3}^{-}\right)+P_{1}^{-}\left(P_{3}^{+}-P_{3}^{-}\right) C\right) \varphi \\
& =\left(P_{1}^{+}(I-C)+P_{1}^{-}\left(P_{3}^{+}-P_{3}^{-}\right)(I+C)\right) \varphi .
\end{aligned}
$$

We took advantage of the obvious commutative equalities $P_{k}^{ \pm} C=C P_{k}^{\mp}$. Conversely,

$$
\varphi=\left(P_{1}^{+} P_{3}^{+}-C P_{1}^{+} P_{3}^{-}\right) F=\left(P_{1}^{+} P_{3}^{+}-P_{1}^{-} P_{3}^{+} C\right) F .
$$

Let us denote

$$
u=P_{1}^{+} P_{3}^{+}-P_{1}^{-} P_{3}^{+} C \quad \text { and } \quad u^{-1}=P_{1}^{+}(I-C)+P_{1}^{-}\left(P_{3}^{+}-P_{3}^{-}\right)(I+C) .
$$

Then

$$
N=u^{-1} R u
$$

where

$$
R=P_{1}^{+}\left(i \partial_{t}+D\right)+P_{1}^{-}\left(-i \partial_{t}+D\right)-m M^{i_{2}}
$$

is the operator of equation (11). Of course, equality (13) can be verified also by a straightforward calculation.

\section{Biquaternionic linear bag model}

Now let us establish the corresponding biquaternionic analog to equation (2). We should find out what happens with the function $\varphi=u \mathcal{A}[\Phi]$ when the original function $\Phi$ is time-harmonic. It was shown in [10] that if $\Phi=q(x) \mathrm{e}^{\mathrm{i} \omega t} \quad(\omega \in \mathbf{R})$, then

$$
\begin{aligned}
\mathcal{A}[\Phi] & =P_{3}^{+} \mathcal{A}[q(x)] \mathrm{e}^{\mathrm{i} \omega t}+\mathrm{P}_{3}^{-} \mathcal{A}[\mathrm{q}(\mathrm{x})] \mathrm{e}^{-\mathrm{i} \omega t} \\
& =\mathrm{e}^{\mathrm{i} \omega \mathrm{t}} \mathrm{P}_{3}^{+} \mathcal{A}[\mathrm{q}(\mathrm{x})]+\mathrm{e}^{-\mathrm{i} \omega \mathrm{t}} \mathrm{P}_{3}^{-} \mathcal{A}[\mathrm{q}(\mathrm{x})]
\end{aligned}
$$

Applying the transform $u$ to this equality we get

$$
u \mathcal{A}[\Phi]=\mathrm{e}^{\mathrm{i} \omega t} \mathrm{P}_{1}^{+} \mathrm{P}_{3}^{+} \mathcal{A}[\mathrm{q}(\mathrm{x})]-\mathrm{C}\left(\mathrm{e}^{-\mathrm{i} \omega t} \mathrm{P}_{1}^{+} \mathrm{P}_{3}^{-} \mathcal{A}[\mathrm{q}(\mathrm{x})]\right)=\mathrm{e}^{\mathrm{i} \omega \mathrm{t}} \mathrm{u} \mathcal{A}[\mathrm{q}(\mathrm{x})]
$$

We obtain that the time-harmonic function $\Phi$ is transformed into the time-harmonic function $\varphi=e^{i \omega t} \rho(x)$. Moreover,

$$
R\left(\mathrm{e}^{\mathrm{i} \omega \mathrm{t}} \rho(\mathrm{x})\right)=-\omega\left(P_{1}^{+}-P_{1}^{-}\right)\left(\mathrm{e}^{\mathrm{i} \omega \mathrm{t}} \rho(\mathrm{x})\right)+D\left(\mathrm{e}^{\mathrm{i} \omega \mathrm{t}} \rho(\mathrm{x})\right)-m M^{i_{2}}\left(\mathrm{e}^{\mathrm{i} \omega \mathrm{t}} \rho(\mathrm{x})\right) .
$$


Therefore for the amplitude $\rho$ from (11) we have the equation

$$
D \rho-i \omega \rho i_{1}-m \rho i_{2}=0
$$

that is, the $I H(\boldsymbol{C})$-valued function $\rho$ satisfies equation (4) with the parameter $\alpha=$ $-\left(i \omega i_{1}+m i_{2}\right)$. Thus equation (4) with the above parameter $\alpha$ is equivalent to equation (2). If $q$ is a solution of equation (2), then $\rho=u \mathcal{A}[q]$ satisfies equation (4) and vice versa, if $\rho \in \operatorname{Ker} D_{\alpha}$ with $\alpha=-\left(i \omega i_{1}+m i_{2}\right)$, then $q=\mathcal{A}^{-1} u^{-1}[\rho]$ is a solution of equation (2).

Consequently, we are able to write down Theorem 1 and Theorem 2 for solutions of equation (2).

Let us denote

$$
\mathbb{K}_{\omega, m}:=\mathcal{A}^{-1} u^{-1} K_{\alpha} u \mathcal{A}= \begin{cases}\mathcal{A}^{-1} u^{-1}\left(\Pi^{+} K_{\xi}+\Pi^{-} K_{\zeta}\right) u \mathcal{A} & \text { if } \omega^{2} \neq m^{2} \\ \mathcal{A}^{-1} u^{-1}\left(K_{0}-M^{\alpha} V_{0}\right) u \mathcal{A} & \text { if } \omega^{2}=m^{2}\end{cases}
$$

(it is clear that the inclusion $\alpha \in \mathcal{S}$ is equivalent to the equality $\omega^{2}=m^{2}$ ) and, for $x \in \Gamma$,

$$
\left(\boldsymbol{S}_{\omega, m} q\right)(x)=2\left(\mathbb{K}_{\omega, m} q\right)(x) \quad \text { and } \quad \not P_{\omega, m}=\frac{1}{2}\left(I+\boldsymbol{S}_{\omega, m}\right)
$$

(the integrals here exist in the sense of Cauchy principal values). Then, for example, an analog of the Cauchy integral formula for time-harmonic massive spinor fields has the following form.

Theorem 4. Let $q \in C^{1}(\tilde{\Omega}) \cap C(\bar{\Omega}) \cap \operatorname{Ker} D_{\omega, m}(\tilde{\Omega})$. Then $q(x)=\left(K_{\omega, m} q\right)(x)$ for all $x \in \tilde{\Omega}$.

Proof. From the hypothesis of the theorem it follows that

$$
\rho:=u \mathcal{A}[q] \in C^{1}(\Omega) \cap C(\bar{\Omega}) \cap \operatorname{Ker} D_{\alpha}(\Omega)
$$

with $\alpha=-\left(i \omega i_{1}+m i_{2}\right)$. Then $\rho=K_{\alpha} \rho$ in $\Omega$. That is, $u \mathcal{A}[q]=K_{\alpha} u \mathcal{A}[q]$. Thus $q=\mathcal{A}^{-1} u^{-1} K_{\alpha} u \mathcal{A}[q]$

With a reasoning along similar lines we are able to obtain the analogs of all points of Theorem 1. Here we restrict ourselves to the analog of Theorem 2.

Theorem 5. Let be given a function $p: \tilde{\Gamma}=\partial \tilde{\Omega} \rightarrow \boldsymbol{C}^{\mathbf{4}}$ satisfying the Hölder condition. In order for $p$ to be a boundary value of a solution $q$ of equation (2) in $\tilde{\Omega}$ it is necessary and sufficient that $p=\mathbb{P}_{\omega, m} p$ on $\tilde{\Gamma}$. Moreover, if this condition is satisfyed, then $q=\mathbb{K}_{\omega, m} p$ is the above mentioned solution of equation (2).

The proof is analogous to that of Theorem 4 with the use of Theorem 2.

Now let us obtain the biquaternionic analog of the boundary condition (3). First of all we have to apply the mapping $\mathcal{A}$ to both sides of (3). Let us multiply (3) from the left by $\dot{\gamma}_{0}$. Then the equality

$$
\mathcal{A}\left[\left.\left(\sum_{k=1}^{3} \gamma_{o} \gamma_{k} \tilde{n}_{k} q\right)\right|_{\bar{\Gamma}}=\left.\left(i \gamma_{0} q\right)\right|_{\bar{\Gamma}}\right]
$$


is equivalent to

$$
\left.\left(\mathcal{A}\left[\sum_{k=1}^{3} \gamma_{0} \gamma_{k} \tilde{n}_{k} q\right]\right)\right|_{\Gamma}=\left.\left(\mathcal{A}\left[i \gamma_{0} q\right]\right)\right|_{\Gamma} .
$$

From the right part we have $\mathcal{A}\left[i \gamma_{0} q\right]=-(\mathcal{A}[q])^{*} i_{3}$ (see Lemma 1). From the left part, changing the normal to $\tilde{\Gamma}$ by that to $\Gamma$ and using Lemma 1 , we obtain

$$
\left.\left(\mathcal{A}\left[\sum_{k=1}^{3} \gamma_{0} \gamma_{k} \tilde{n}_{k} q\right]\right)\right|_{\Gamma}=\left.(i \vec{n} \mathcal{A}[q])\right|_{\Gamma}
$$

Thus, equality (3) turns into $i \vec{n} \mathcal{A}[q]+C \mathcal{A}[q] i_{3}=0$ on $\partial \Omega$ or into the equivalent form

$$
\frac{1}{2}\left(I+\vec{n} i M^{i_{3}} C\right) \mathcal{A}[q]=0 \quad \text { on } \Gamma \text {. }
$$

Let us denote

$$
Q^{ \pm}:=\frac{1}{2}\left(I \pm \vec{n} C\left(P_{3}^{+}-P_{3}^{-}\right)\right)=\frac{1}{2}\left(I \mp \vec{n} i M^{i_{3}} C\right)
$$

Then (15) can be rewritten as

$$
Q^{-} \mathcal{A}[q]=0 \quad \text { on } \Gamma \text {. }
$$

It is easy to verify that the operators $Q^{+}$and $Q^{-}$are mutually complementary projectors on a set of $I H\left(\boldsymbol{C}^{*}\right)$-valued functions which is invariant with respect to them. Then the operators $S^{ \pm}=u Q^{ \pm} u^{-1}$ are also mutually complementary projectors.

It remains to obtain the explicit form of the operators $S^{ \pm}$. From (13) it follows that $m i C M^{i_{3}}=u^{-1} m M^{i_{2}} u$. That is, $u\left(i M^{i_{3}} C\right) u^{-1}=M^{i_{2}}$. Then

$$
S^{ \pm}=\frac{1}{2}\left(I \mp \vec{n} u\left(i M^{i_{3}} C\right) u^{-1}\right)=\frac{1}{2}\left(I \mp \vec{n} M^{i_{2}}\right)
$$

and, for $\rho=u \mathcal{A}[q]$, the equality (16) turns into the boundary condition

$$
S^{-} \rho=0 \text {. on } \Gamma \text {. }
$$

Thus the boundary value problem $(14),(17)$ is equivalent to the bag model (2), (3), and the equivalence is determined by the bijection $u \mathcal{A}$.

It is clear that condition (17) can be rewritten in the form $\rho=S^{+} \rho$ on $\Gamma$. Thus from Theorem 2 a simple criterion of solvability for the boundary-value problem (14), (17) follows.

Theorem 6. The problem (14),(17) for a given function $\rho \in C^{0, \varepsilon}(\Gamma) \quad(0<\varepsilon \leq$ $1), \rho: \Gamma \rightarrow \mathbb{H}(\boldsymbol{C})$ is solvable if and only if.

$$
\rho=P_{a} \rho=S^{+} \rho \text { on } \Gamma \text {. }
$$

In this way the bag model (2), (3) is reduced to the boundary equation (18).

Conclusions. The biquaternionic analysis allows to develope enough methods for the treatment of boundary-value problems relative to the operator $D_{\alpha}(a \in \mathbb{H}(\boldsymbol{C}))$. Some of the most simple problems were studied in $[8,9,18,19]$. The above considerations show that all results in this direction are applicable to the analysis of quark models. Moreover, it is imperative to build up a systematic solvability and Fredholm theory in connection with analogs of Hilbert and Riemann problems for the operator $D_{\alpha}$. 


\section{References}

'1] Beresin, A. V., Kurochkin, Yu. A. and E. A. Tolkachev: Quaternions in the Relativistic Physics (in Russian). Minsk: Nauka y Tekhnika 1989.

[2] Beresin, A. V., Tolkachev, E. A. and F. I. Federov: Lorentz transformations and the equations for spinor quaternions (in Russian). Dokl. Akad. Nauk BSSR 24 (1980)4, 308 $-310$.

[3] Bizadse, A. V.: Foundations of the Theory of Analytic Functions of a Complex Variable (in Russian). Moscow: Nauka 1984.

[4] Close, F.: An introduction to Quarks and Partons (in Russian). Moscow: Mir 1982.

[5] Dzhuraev, A. D.: Method of Singular Integral Equations (in Russian). Moscow: Nauka 1987.

[6] Gürlebeck, K. and W. Sprößig: Quaternionic Analysis and Elliptic Boundary Value Problems. Berlin: Akademie - Verlag 1989.

(7) Janushauskas, A.: Multivariate Elliptic Systems with Varying Coefficients (in Russian). Vilnus: Mokslas Publ. 1990.

[8] K'ravchenko, V. V.: Integral Representations of Biquatemionic Hyperholomorphic Functions and Their Applications (in Russian). Thesis. Odessa: State University 1993.

[9] Kravchenko, V. V.: Generalized holomorphic vectors and $\alpha$-hyperholomorphic function theory. J. Nat. Geom. 6 (1994) (to appear).

[10] Kravchenko, V. V.: Quaternion-valued integral representations for time-harmonic electromagnetic and spinor fields. Dokl. Akad. Nauk Russia (to appear).

[11] Kravchenko, V. V. and M. V. Shapiro: On a generalized system of Cauchy-Riemann equations with a quaternionic parameter (in Russian). Dokl. Akad. Nauk Russia 329 (1993), 547 - 549. Engl. transl. in: Dokl. Russ. Acad. Sci. 47 (1993)2, 315 - 319.

[12] Kravchenko, V. V. and M. V. Shapiro: Helmholtz operator with a quaternionic wave number and associated function theory. In: Deformations of Mathematical Structures. Part II: Hurwitz-Type Structure and Applications to Surface Physics (ed.: J. Lawrynowicz). Dordrecht: Kluwer Acad. Publ. 1994, pp. 101 - 128.

[13] Kravchenko, V. V. and M. V. Shapiro: Helmholtz operator with a quaternionic wave number and associated function theory. Part II: Integral representations. Acta Appl. Math. 32 (1993), 243 - 265.

[14] Krupnik, N. Ya: Banach Algebras with Symbol and Singular Integral Operators (in Russian). Kishinev: Shtiinca 1984.

[15] Litvinchuk, G. S.: Boundary Value Problems and Singular Integral Equations with Shift (in Russian). Moscow: Nauka 1977.

[16] Liede, Huang: The existence and uniqueness theorems of the linear and nonlinear RiemannHilbert problems for the generalized holomorphic vector of the second kind. Acta Math. Sci. (Engl. ed.) 10 (1990), 185 - 199.

[17] Mostepanenko, V. M. and N. N. Trunov: Casimir's Effect and Its Applications (in Russian). Moscow: Energoatomizdat 1990.

[18] Obolashvili, E. I.: Spatial generalized holomorphic vectors (in Russian). Dif. uravneniya 11 (1975), $108-115$.

[19] Shapiro, M. V.: On analogies of the Riemann boundary value problem for a class of hyperholomorphic functions. In: Integral equations and boundary value problems (eds.: Zhao Zhen and Wen Guochun). Singapore: World Sci. 1991, pp. 184 - 188. 
[20] Shapiro, M. V. and N. L. Vasilevski: Quaternionic $\Psi$-hyperholomorphic functions, singular operators with quaternionic Cauchy kernels and analogues of the Riemann boundary value problem. Preprint. Mexico City: Dep: of Math., CINVESTAV del IPN, Preprint Nr. 102 (1992), 1 - 75. To appear as a two-part article in: Complex Variables, Theory and Appl. (1994).

[21] Thomas, A. W.: Chiral symmetry and the bag model. A new starting point for nuclear physics. Adv. Nucl. Phys. 13 (1984), 1 - 137.

Received 16.03.1994 